\title{
Evaluation of Lower Limb Arterial Angioplasty using Doppler Ultrasound in Diabetic Patients
}

\author{
M.M.Refaat ${ }^{1}$, O.T.Galal ${ }^{2}$ and A.H.Elsaied ${ }^{3}$
}

${ }^{1}$ Professor \& Head of Radiodiagnosis Dept., Faculty of Medicine, Benha Univ., Benha,Egypt

${ }^{2}$ Lecturer at Radiodiagnosis Dept., Faculty of Medicine, Benha Univ., Benha, Egypt

${ }^{3}$ Radiodiagnosis Dept., National Institute of Diabetes, Egypt

E-Mail: alihosny201116@gmail.com

\begin{abstract}
Duplex scanning considered the imaging modality of choice for detection and follow up of patients suffering from Peripheral arterial disease as it is inexpensive, radiation-free and provides both anatomic and hemodynamic information about the lesion. Lower limb arterial angioplasty is now has the greatest role in management of patients suffering from lower limb ischemia.
\end{abstract}

Keywords: Peripheral arterial disease, Llower limb arterial angioplasty, Duplex scanning.

\section{Introduction}

Peripheral arterial disease (PAD) affects almost $12 \%$ of the general population and is responsible for substantial healthcare costs. PAD primarily results in a decreased functional capacity and deterioration in quality of life and is associated with an increased risk of limb amputation, myocardial infarction, stroke, and death [1].

Two-thirds to three-fourths of patients initially presenting with intermittent claudication (IC) symptoms will remain stable for several years after the initial diagnosis, whereas the remaining one-third to one-fourth will show progressive disease, but only $1-5 \%$ of the PAD population will eventually undergo amputation. Patients suffering from PAD typically present with symptoms of IC or Critical limb ischemia [2]. Patients with CLI are at high risk of major amputation, ranging from $10-40 \%$ at one year after their diagnosis, with mortality approaching $25 \%$ [3].

The commonest indication for duplex ultrasound in lower extremity arterial disease is to identify potential percutaneous intervention in patients with intermittent claudication. It establishes the diagnosis, the anatomical site of disease, and can define the severity of a focal stenosis. It can help decide whether to proceed with angiography, and aid the planning of the optimum treatment approach to a lesion. The goals of treatment for patients with CLI (critical limb ischemia) include limb salvage, as well as reduction of major adverse cardiovascular events. In the past decade, endovascular techniques have been increasingly employed for prevention of major amputation among patients with CLI. This endovascular first approach has been accompanied by the development of many new technical approaches for treatment of peripheral arterial disease (PAD) [4].

\section{Diagnostic criteria of PAD}

Patients suffering from PAD typically present with symptoms of IC (intermittent claudication) or CLI. IC is defined as an intermittent cramping pain during walking caused by an inadequate supply of blood to the musculature of the lower limb. IC typically develops on exertion and is relieved at resting conditions. CLI is the final clinical manifestation of PAD and is typically characterized by either chronic ischemic rest pain and/or ischemic tissue loss of the limb (ulcers or gangrene) attributable to objectively proven arterial occlusive disease [5]. CLI is defined by an ankle systolic pressure of $50 \mathrm{mmHg}$ or less. In case of incompressible arteries at the ankle, a toe systolic pressure of $30 \mathrm{mmHg}$ or less can be used [6]. Resting ABI measurements are to be classified and reported as >1.4 (non-compressible values), 1.0-1.4 (normal), 0.90-0.99 (borderline), and <0.9 (abnormal) according to the Ankle Brachial Index Collaboration [7]. An ABI $<0.9$ is considered as highly sensitive $(95 \%)$ and specific $(100 \%)$ for the diagnosis of PAD [8]. Measurements of Toe pressure (critical level $<30 \mathrm{mmHg}$ ) are suggested in case of diabetic patients with ulcers both for baseline evaluation and for assessment of response to revascularization [9]. The ankle peak systolic velocity is defined as the mean of peak systolic velocity of the anterior tibial and posterior tibial arteries. Normally it is above $75 \mathrm{~cm} / \mathrm{sec}$, if it is below $25 \mathrm{~cm} / \mathrm{sec}$ it is an index of critical limb ischemia [10].

\section{Imaging of PAD}

\subsection{Doppler imaging of PAD}

The Doppler measurements show an increase of velocity up to a maximum near the point of critical stenosis, followed by steady decline down to zero velocity at occlusion. Velocity begins to increase noticeably at about $30 \%$ diameter reduction, and then increase rapidly at above $50 \%$. Flow remains substantially 
unaffected until about $75-80 \%$ diameter reductions. After about $85 \%$, the critical point has been exceeded, both velocity and flow drop. There are four particular Doppler features of disturbed flow:
1- Increased jet velocity.

2-Spectral broadening.

3-Simultaneous forward and reverse flow (turbulence).

4-Fluctuations of flow velocity with time [11].

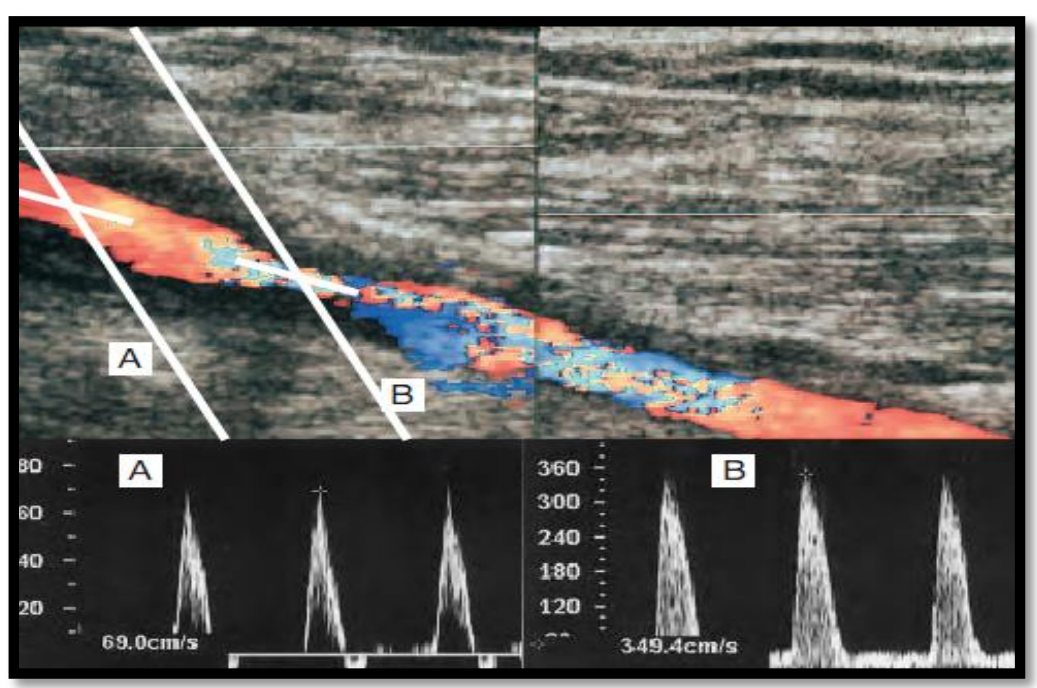

Fig (1) Superficial femoral artery stenosis is assessed using spectral Doppler. A- Measurement of the PSV just proximal to the stenosis. B- Measurement of the PSV across the stenosis indicating severe stenosis [12].

\subsection{Angioplastic imaging}

Multidisciplinary decision-making for treatment must take into account the patient's clinical symptoms, the anticipated lifeexpectancy, morphological classification of the femoropopliteal atherosclerosis, anatomical challenges, renal failure, contrast allergy, and the availability of vein conduits. In patients with limb-threatening ischemia and a life-expectancy of $<2$ years or in the absence of a suitable vein conduit, percutaneous transluminal angioplasty is recommended as the first-line treatment regardless of the anatomical extent of disease. Patients with heavily calcified femoropopliteal lesions and a life-expectancy of $>2$ years may be first considered for vein bypass surgery [1].

The main steps of angioplasty could be summarized in three steps; how to gain an access to the target vessel, how to cross the lesion and reentry of the main lumen with balloon dilatation or stent placement according to the number, type of lesion, degree and the hemodynamic effect of the lesion. The choice between antegrade and contralateral retrograde access will depend on body habitus and the presence of concomitant inflow iliac disease. Any significant inflow disease must be treated before treating SFA and popliteal lesions [13].

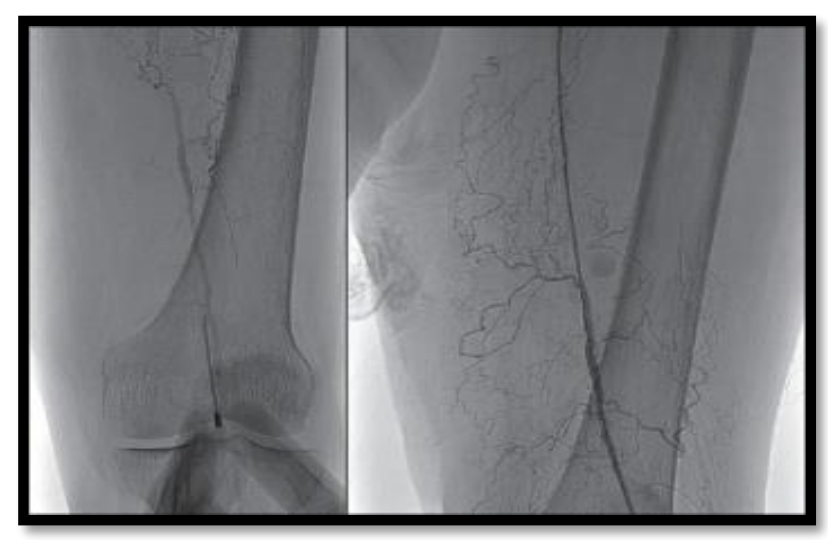

Fig (2) Roadmap technique in inserting distal access; the right one: Needle headed the popliteal artery, the 
left; angiography did after the popliteal sheath inserted [14].

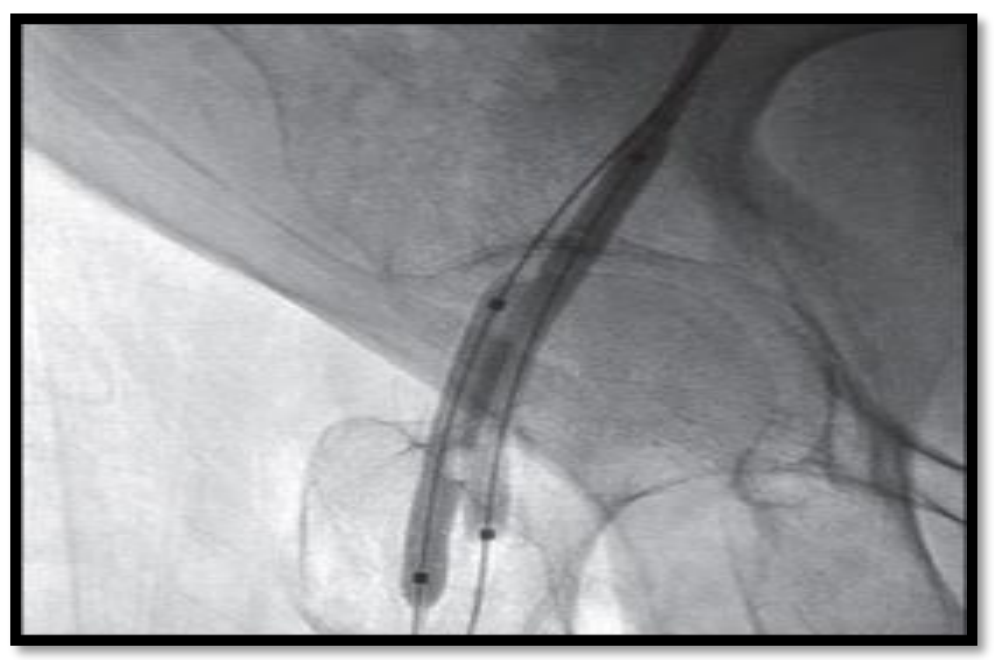

Fig (3) Double balloon technique illustrating two different sized balloon one from different access (one proximal and the other distal) inflated at the same time to crush the atherosclerotic plaque in-between [14].

\section{Aim of the study}

Doppler assessment for lower limb angioplasty in diabetic patients with peripheral arterial diseases.

\section{Patients and methods \\ 5.1 Patient selection}

This study was conducted at the Radiology department- National Institute of Diabetes Cairo Governorate - Ministry of health in the time frame from April 2016 till April 2018, included 25 clinically diagnosed PAD patients who referred from Vascular Department and outpatient clinics. They were 11 females and 14 males. All patients have done Doppler study pre and post angioplasty.

\section{Patient inclusion criteria}

1- Incapacitating claudication interfering with the work or life style, severe claudication, cannot complete treadmill exercise and ankle pressure after exercise was less than $50 \mathrm{mmHg}$.

2-Critical limb ischemic with rest pain, resting ankle pressure was less than $40 \mathrm{mmHg}$, minor tissue loss, non-healing ulcer or focal gangrene and major tissue loss.

3-Patients with lesions, A (single stenosis $<1 \mathrm{~cm}$ in length), B [Multiple focal $(<1 \mathrm{~cm})$ stenoses of the tibial or peroneal arteries (including up to 2 focal stenoses at the tibial trifurcation) and short tibial or peroneal stenoses in conjunction with femoropopliteal disease], $\mathrm{C}$ (longer stenoses 1-4 cm and occlusions 1-2 $\mathrm{cm}$ as well as extensive stenoses at the tibial trifurcation and D (occlusions longer than 2 $\mathrm{cm}$ and diffusely diseased tibial vessels).

\section{Patient exclusion criteria}

1- Proximal aorto-iliac disease.

2-Amputated foot or leg below knee.

3-Known intolerance to study medications or contrast agents.

\subsection{Technique}

All of the 25 patients had lower limb arterial Doppler study in National Diabetic Institute. The patients were scheduled for duplex scanning before intervention, immediately 1 to 7 days after intervention. At first the patient lie in supine position then arterial tree is scanned from CFA down to distal tibial arteries or by reverse.

Color and spectral Doppler scan were done by the same operator .A day prior to the angioplasty all subjects were examined with Bmode, color and duplex US using a $7-12-\mathrm{MHz}$ linear array transducer for infra-inguinal arterial assessment, sometimes a $2-5-\mathrm{MHz}$ convex array transducer used for obese patients and for assessment of the distal part of the superficial femoral artery in the Hunter area in some cases. We detect the following:

1- Anatomical site, occlusion or stenosis (single or multiple).

2-Ankle peak systolic velocity(APSV)

3-Ankle-brachial index (ABI).

4-Toe brachial index (TBI).

The patients were admitted one day before or on the day of the procedure, a loading dose of clopidogrel $300 \mathrm{mg}$ was given the night of 
the procedure, both groins were prepared using an antiseptic solution (povedin). All equipment were checked including monitors, connections, light system, catheters, wires, sheaths, different sized ballons, stents and emergency kits. The patient lies in the supine position and a local anesthetic is given (xylocaine $2 \%$ ). We used an ipsilateral or contralateral antegrade femoral access following anatomical localization of the CFA (common femoral artery) in all patients.

\subsection{Data analysis and Statistical data display}

To obtain diagnostic values, we shall measure sensitivity, specificity, positive and negative predictive values of the analyzed data using commercially available PC-based software package (SPSS).

\section{Results}

The results recorded in table (1) shows that the study included 25 patients, 14 males and 11 females, where males represented $56 \%$ and females represented $44 \%$.

Table (2) shows risk factors for peripheral arterial disease as following: Diabetic 25 patients represents $100 \%$, HTN 16 patients represents 64\%, Cardiac 11 patients represents $44 \%$, CVS 4 patients represents $16 \%$ and Smokers 13 patients represents $52 \%$.

Table (3) shows Clinical presentation of all patients as following: Claudication 8 patients represents $32 \%$, rest pain 2 patients represents $8 \%$ and ischemic ulcer 15 patients represents $60 \%$.

Table (4) shows type of lesion as following: Stenosis 11 patients represents $44 \%$, occlusion 10 patients represents $40 \%$ and Mixed 4 patients represents $16 \%$.

Table (5) shows comparison between pre and post procedural status regarding the ankle peak systolic velocity (APSV), the ankle brachial index (ABI) and toe brachial index (TBI).

Table (6) shows the overall result of the study: twenty five patients underwent an infrainguinal angioplasty, twenty three of them had a successful procedure $(92 \%)$ and two had a partial successful procedure $(8 \%)$.

Table (1) Distribution of patients according to sex.

\begin{tabular}{lcc}
\hline & Number & Percentage \\
\hline Males & 14 & $56 \%$ \\
Females & 11 & $44 \%$ \\
\hline
\end{tabular}

Table (2) Risk factors of lower limb ischemia

\begin{tabular}{lcc}
\hline & Number & Percentage \\
\hline Diabetic & 25 & $100 \%$ \\
HTN & 16 & $64 \%$ \\
Cardiac & 11 & $44 \%$ \\
CVS & 4 & $16 \%$ \\
Smoker & 13 & $52 \%$ \\
\hline
\end{tabular}

Table (3) Clinical presentation of all patients

\begin{tabular}{lcc}
\hline & Number & Percentage \\
\hline Claudication & 8 & $32 \%$ \\
Rest pain & 2 & $8 \%$ \\
Ulcers & 15 & $60 \%$ \\
\hline
\end{tabular}

Table (4) Type of lesion

\begin{tabular}{lcc}
\hline & Number & Percentage \\
\hline Stenosis & 11 & $44 \%$ \\
Occlusion & 10 & $40 \%$ \\
Mixed & 4 & $16 \%$ \\
\hline
\end{tabular}


Table (5) Comparison between pre and post procedural status regarding the ankle peak systolic velocity (APSV), the ankle brachial index (ABI) and toe brachial index (TBI)

\begin{tabular}{lcccccc}
\hline Mean & $\begin{array}{c}\text { Standard } \\
\text { Deviation }\end{array}$ & Median & Minimum & Maximum & P value \\
\hline ABI pre & 0.585 & 0.11 & 0.60 & 0.40 & 0.72 & $<0.01$ \\
ABI & 0.848 & 0.05 & 0.85 & 0.80 & 0.90 & \\
APSV & 22.85 & 12.39 & 22.0 & 7.00 & 50.00 & $<0.002$ \\
APSV & 46.92 & 21.65 & 40.0 & 22.00 & 94.00 & \\
TBI pre & 0.557 & 0.05 & 0.55 & 0.50 & 0.68 & $<0.01$ \\
TBI & 0.671 & 0.04 & 0.67 & 0.59 & 0.74 & \\
\hline
\end{tabular}

Table (6) The overall result of the study

\begin{tabular}{lccc}
\hline & Count & $\%$ & P value \\
\hline Success & 23 & $92 \%$ & \\
Partial success & 2 & $8 \%$ & $<0001$ \\
\hline
\end{tabular}

\section{Discussion}

Peripheral arterial disease affects almost $12 \%$ of the general population and is responsible for substantial healthcare costs. PAD primarily results in a decreased functional capacity and deterioration in quality of life and is associated with an increased risk of limb amputation, myocardial infarction, stroke, and death [1].

Percutaneous angioplasty and stenting of the superficial femoral and popliteal artery is the proposed treatment of choice in the majority of patients with IC or CLI on the basis of its reduced perioperative morbidity and mortality, and reduced in-hospital stay [15].

To date, several new technologies, such as bare metal stents made from nitinol, drugeluting stents (DES), covered stents, and drugcoated balloons (DCB), have emerged with the aim to improve long-term patency outcomes following angioplasty of the femoral and popliteal arteries [16].

Over the last three decades, color duplex ultrasonography has gained acceptance and established itself as a fundamental component of diagnostic evaluation and management of arterial disease. Duplex technology is suitable for directing endovascular interventions and revascularization, permitting not only the identification of disease, but also assessment of its response to intervention [17].

In the current study, we used color duplex ultrasonography as a diagnostic study for assessment of the pre interventional status of the lower limb arterial tree and detection of the site, type,degree and the hemodynamic effect of any stenosis or occlusion.
Among our study, we have 25 patients unlike Cho Set al., 2006, Tan M et al., 2010 [18] and Zou J 2012 [19]. 14 patients are males $(56 \%)$ and 11 patients are females $(44 \%)$.

All the patients are diabetics (100\%) the same as Tan M et al [18], (2010), 16 patients are hypertensive (64\%), eleven patients are cardiac (44\%), 4 patients with history of cerebrovascular stroke $(16 \%)$ and 13 patients are smoker $(52 \%)$.

Fifteen of them presented by non-healing ulcers $(60 \%)$ in agreement with Kudo $\mathrm{T}$ et al (2005) [20] and not similar to Ghoneim B et al (2014) [21], and Lida et al (2012) [22].

Eight patients presented with incapacitating intermittent claudication $(32 \%)$ while two patients presented with rest pain $(8 \%)$ with disagreement to Ghoneim B et al (2014) [21], Lida et al (2012) [22], Tan M et al (2010) [18] which is not similar to Kudo et al (2005) [20].

Post interventional color duplex ultrasound was done to the twenty five patients for assessment of the technical success of the procedure by assessing the ABI, APSV,TBI and the percentage of remaining stenosis if present.

We found that the there is sufficient elevation of above parameter after angioplasty.

The overall complete technical success in our study was $92 \%$ with two partial success patient $(8 \%)$, while the technical success for (Ghoneim B et al., 2014[21]) was 96.8\%, (Dosluoglu et al., 2009[23]) was 97\% and (Lida et al., 2012[22]) was 94\%.

As regards these two patients with partial success, the pre interventional duplex assessment of these patients revealed total occlusion of the distal third of the SFA and the 
supragenicular segment of the popliteal artery, they underwent balloon dilatation of the diseased segments but the post procedural duplex scan revealed a significant stenosis resulting in about $57 \%$ and $60 \%$ diameter reduction.

\section{Conclusion}

To sum up the endovascular approach is recommend recommended as the first-line treatment in the majority of lesions of the superficial femoral and/or popliteal arteries. Recanalization can be achieved using either intraluminal or subintimal techniques. In more challenging cases, more complex techniques (i.e., SAFARI technique) and advanced devices (re-entry devices, atherectomy catheters) may be required to achieve crossing of the occluded segment. However, preservation of long-term patency remains a challenge, mainly due to progressive neointimal hyperplasia and vascular restenosis.

The uses of Duplex ultrasound is considered the best imaging modality for early diagnosis and follow up of patients suffering from lower limb ischemia as it is reliable, inexpensive, radiation-free and provides both anatomic and hemodynamic information about the degree of any lesion.

\section{References}

[1] T.W.Rooke, A.T.Hirsch, S.Misra et al., American College of Cardiology Foundation/American Heart Association focused update of the guideline for the management of patients with peripheral artery disease (updating the 2005 guideline). J Vasc Surg,Vol. 54(5), pp.e32e58, 2011.

[2] M.J.Pentecost, M.H.Criqui, G.Dorros, et al., Guidelines for peripheral percutaneous transluminal angioplasty of the abdominal aorta and lower extremity vessels: A statement for health professionals from a special writing group of the Councils on Cardiovascular Radiology, Arteriosclerosis, Cardio-Thoracic and Vascular Surgery, Clinical Cardiology, and Epidemiology and Prevention, the American Heart Association. Circulation, vol.89, pp.511531, 1994.

[3] A.M.Abu Dabrh, M.W.Steffen, C.Undavalli, et al., The natural history of untreated severe or critical limb ischemia. J Vasc Surg, vol.S0, pp.741-521, 2015.

[4] S.Cassese, R.A.Byrne, I.Ott et al., Paclitaxelcoated versus uncoated balloon angioplasty reduces target lesion revascularization in patients with femoropopliteal arterial disease: a meta-analysis of randomized trials. Circ Cardiovasc Interv, Vol. 5(4), pp.582-589, 2012.

[5] L.Norgren, W.R.Hiatt, J.A.Dormandy et al., Inter-Society Consensus for the Management of Peripheral Arterial Disease (TASC II). Eur J Vasc Endovasc Surg,vol. 33(Suppl 1),pp.S1-S75, 2007.

[6] D.Slovut and T.M.Sullivan, Critical limb ischemia: medical and surgical management. Vasc Med, vol.13 (3), pp.281-291, 2008.

[7] F.G.Fowkes, G.D.Murray, I.Butcher et al., Ankle brachial index combined with Framingham Risk Score to predict cardiovascular events and mortality: a metaanalysis. JAMA, vol.300 (2), pp.197-208, 2008.

[8] S.Allaqaband, R.Kirvaitis, F.Jan, T.Bajwa., Endovascular treatment of peripheral vascular disease. Curr Probl Cardiol, vol. 34(9), pp.359-476, 2009.

[9] R.Ladurner, M.Kuper, I.Konigsrainer et al, Predictive value of routine transcutaneous tissue oxygen tension (tcpO2) measurement for the risk of non-healing and amputation in diabetic foot ulcer patients with nonpalpable pedal pulses. Med Sci Monit, vol.16 (6), pp.CR273-CR277, 2010.

[10] R.Bishara , W.Taha, I. Akladious, et al., Ankle Peak Systolic Velocity: New Parameter to Predict Nonhealing in Diabetic Foot Lesions SAGE J, vol. 17 no. 5,pp. 264-268, 2009.

[11] P.N.Burns, Hemodynamics. In Taylor KJW, Burns PN, Wells PN (ed.): Clinical application of Doppler ultrasound, vol.2 (2nd edition). Raven Press, New York, pp. 35-52, and 1995.

[12] A.Thrush and T.Hartshorn, Peripheral vascular ultrasound. How, Why and When,vol.9(2nd edition), Elsevier Churchill Livingstone; pp. 111-133, 2005.

[13] F.Fanelli, P.Lucatelli, M.Allegritti, et al., Retrograde popliteal access in the supine patient for recanalization of the superficial femoral artery: initial results. J Endovasc Ther, vol.18 (4), pp.503-509, 2011.

[14] M.Hagag, A.Tawfik, U.Lotfi et al., Overcoming Difficult Cnic Total Occlusion: Increasing the Applicability of Endovascular Intervention to Patients with Challenging Re - entry: Double Balloon Technique in Crossing Challenging Chronic Total Occlusions. Indian $\mathrm{J}$ vascular and endovascular Surgery, vol.3, 2, pp. 40-404, 2016.

[15] M.Tendera, V.Aboyans, M.L.Bartelink et al., ESC Guidelines on the diagnosis and treatment of peripheral artery diseases: 
document covering atherosclerotic disease of extracranial carotid and vertebral, mesenteric, renal, upper and lower extremity arteries: the Task Force on the Diagnosis and Treatment of Peripheral Artery Diseases of the European Society of Cardiology (ESC). Eur Heart J, vol.32 (22), pp.2851-2906, 2011.

[16] M.D.Dake, G.M.Ansel, M.R.Jaff et al., Paclitaxel-eluting stents show superiority to balloon angioplasty and bare metal stents in femoropopliteal disease: twelve-month Zilver PTX randomized study results. Circ Cardiovasc Interv, vol.4 (5), pp.495-504, 2011.

[17] P.A.Armstrong, F.E.Parodi, and D.F.Bandyk, Duplex scanning for lower extremity arterial diseases. In Abu Rahma AF., Bandyk DF. (ed.): Noninvasive vascular diagnosis, vol.3 (3rd edition). Springer, London, pp.: 311- 320, 2013.

[18] M.Tan, U.Pua, D.Wong et al., Critical limb ischaemia in a diabetic population from an Asian Centre: angiographic pattern of disease and 3-year limb salvage rate with percutaneous angioplasty as first line of treatment Biomed Imaging Interv J. 10.2349/biij.6.4.e33, 2010.

[19] J.Zou, Y.Xia, H.Yang et al., Hybrid Endarterectomy and Endovascular Therapy in Multilevel Lower Extremity Arterial Disease Involving the Femoral Artery Bifurcation Int Surg,vol.97(1),pp.56-64, 2012.
[20] T.Kudo, F.Chandra and S.Ahn, The effectiveness of percutaneous transluminal angioplasty for the treatment of critical limb ischemia: a 10-year experience J Vasc Surg ,vol.435 (discussion 435),pp.413423435.435, , 2005.

[21] B.Ghoneim, H.Elwan, W.Eldaly et al., Management of Critical Lower Limb Ischemia in Endovascular Era: Experience from 511 Patients national J Angiol, vol.23 (3), pp. 197-206, 2014.

[22] O.Lida, Y.Soga, K.Hirano et al., Midterm outcomes and risk stratification after endovascular therapy for patients with critical limb ischaemia due to isolated below-the-knee lesions. Eur J Vasc Endovasc Surg, vol.43 (3), pp.313-321, 2012.

[23] H.H.Dosluoglu, P.Lall, G.S.Cherr, et al., Superior limb salvage with endovascular therapy in octogenarians with critical limb ischemia. J Vasc Surg, vol.50. Pp.305-316, 2009. 